the illustrations and tables are clear, and the index is comprehensive. It is, however, a little unfortunate that some 15 months should elapse between the symposium and its publication on this side of the Atlantic. This minor criticism apart, this book can be strongly recommended to anyone actively involved in the practice of clinical genetics.

I D Young

\section{Sister Chromatid Exchange}

Progress and Topics in Cytogenetics. Vol 2. Edited by Avery A Sandberg. (Pp xv + 706; figures + tables. $£ 66 \cdot 50$, DM 293.) New York: Alan R Liss. 1982.

Suitably stained chromosome preparations made from cells grown in the presence of bromodeoxyuridine, which is incorporated into newly synthesised DNA in place of thymidine, reveal 'harlequin' chromosomes which display with diagrammatic clarity the difference between chromatids with bromodeoxyuridine substituted and unsubstituted DNA strands. This beautiful demonstration of the operation of semiconservative replication can also be utilised to show, with great precision, exchanges between sister chromatids. A technique of such relative simplicity which comes close to demonstrating molecular events in the structures in which it occurs is a pleasure to use. It is not surprising that workers in laboratories around the world have taken to this technique not only to study cell kinetics but also to investigate the significance of chromosome exchange phenomena in relation to damage and repair of the genetic machinery and the induction of neoplasia.

In this volume Dr Sandberg has gathered some 30 contributions on sister chromatid exchange, 31 if you count one paper on the induction of micronuclei. The contributions are loosely grouped under general headings. 'History and Methodology' is appropriately opened by Zakharov whose introduction in 1972 of bromodeoxyuridine techniques marked the transformation of the study of SCE from the rather limited observations of the behaviour of ring chromosomes (initiated by McClintock in 1938) and Taylor's now classical autoradiographic studies using ${ }^{3} \mathrm{H}$-thymidine, to the much easier, clearer staining techniques currently available. There follows a 'recipe book' review of staining methods, a paper on the use of SCEs to assess the persistence of DNA lesions, and a paper on the induction of micronuclei.

The second section which consists of five papers and is entitled 'Possible Mechanisms of SCE Formation' illustrates well the fascinating and simultaneously frustrating aspect of SCE study. Model building is fun and various models are reviewed which fit more or less well with presently available evidence, but it seems likely that we must await a better knowledge of the molecular events involved before we can properly understand either the cause or the mechanism of the SCE phenomenon.

Most of the rest of the book has its eye firmly fixed on the goal of providing a means for monitoring the mutagenic and carcinogenic effects of environmental agents. A section on 'SCE in Cells and Tissues of Plants, Animals and Humans' attempts to establish baseline frequencies according to species, tissue, and age with due account of the effect of the experimental procedures in SCE induction. SCEs in meiotic cells also receive attention and comparisons are made with meiotic crossing-over.

There are a number of papers reporting on SCE frequency encountered in human disease states with emphasis on those associated with neoplasia or predisposition to neoplasia. Separate sections take the effect of physical and biological agents, of mutagens and carcinogens, of drugs and the value of SCE analysis in Public Health monitoring as their theme.

The papers in this book succeed in covering the main preoccupations of those using this technique and highlight many of the problems and pitfalls of interpretation. They convey well the dilemma of having a technique which clearly demonstrates an effect of DNA damaging agents on the chromosome which is compatible with continued cellular replication but which has so far resisted attempts to correlate it with known mechanisms of DNA repair. This is probably a reflection of the wide spectrum of DNA damage induced by different agents and the multiplicity of repair systems available to the cell, but is a disappointment to those searching for a reliable indicator of carcinogenic potential.

There is little that is new in this book. The papers are essentially of a review type and, inevitably in a collection of presentations by many different people dealing with one observed chromosomal activity, there is much repetition which a single author would be able to avoid, but the book undoubtedly provides a helpful review and bibliography of work and thought in this area at a time appropriate for appraisal of the usefulness of the techniques involved. There is an index too. However, the considerable cost of the book may be a disincentive to many who may wish to use it.

Michael FAed 